\title{
Phase Transitions and Anion Exchange in All-Inorganic Halide Perovskites
}

Julian A. Steele ${ }^{\mathrm{a}, \mathrm{b}^{*}}$, Minliang Lai ${ }^{\mathrm{b}}$, Ye Zhang ${ }^{\mathrm{b}, \mathrm{c}}$, Zhenni Lin ${ }^{\mathrm{c}, \mathrm{d}}$, Johan Hofkens ${ }^{\mathrm{e}, \mathrm{f}}$, Maarten B. J. Roeffaers $^{\mathrm{a}}$, and Peidong Yang ${ }^{\mathrm{b}, \mathrm{c}, \mathrm{d}, \mathrm{g}^{*}}$

${ }^{a}$ cMACS, Department of Microbial and Molecular Systems, KU Leuven, 3001 Leuven, Belgium

${ }^{b}$ Department of Chemistry, University of California, Berkeley, CA 94720

${ }^{\mathrm{c}}$ Materials Sciences Division, Lawrence Berkeley National Laboratory, Berkeley, CA 94720

${ }^{\mathrm{d}}$ Department of Materials Science and Engineering, University of California, Berkeley, CA 94720;

${ }^{\mathrm{e}}$ Department of Chemistry, KU Leuven, 3001 Heverlee, Belgium

${ }^{\mathrm{f}}$ Max Plank Institute for Polymer Research, Mainz, D-55128, Germany

g Kavli Energy Nano Science Institute, Berkeley, CA 94720

\section{TOC Image:}

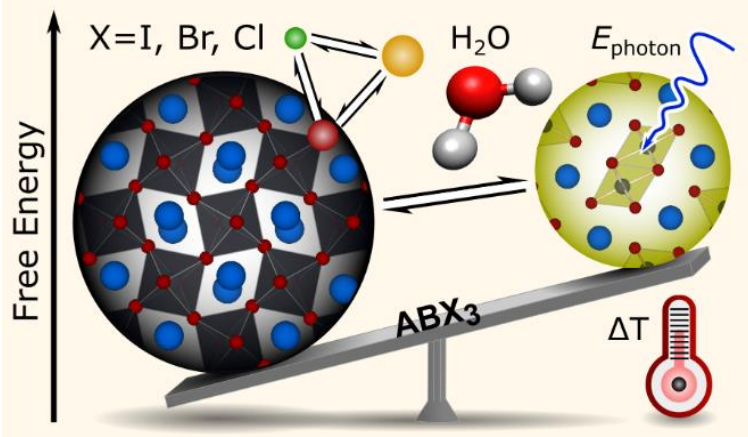




\section{CONSPECTUS:}

A new generation of semiconducting materials based on metal halide perovskites has recently been launched into the scientific spotlight, exhibiting outstanding optoelectronic properties and providing promise for the development of efficient optical devices. As a vivid example, solar cells made from these materials have quickly reached conversion efficiencies exceeding $25 \%$, now on par with well-established technologies, like silicon. Their widespread success is due, in part, to a unique ability to retain high-quality optoelectronic performance while being easily solution-processed into thin films. This feature is what defines them as a brand-new class of optoelectronic materials, with the ability to compete with traditional semiconductors requiring higher processing costs, like the IIIVs or II-IVs. However, the interesting photophysics of metal halide perovskites come with a catch; their soft ionic lattice promotes complex thermal-induced phase transitions and a high capacity for post-synthetic compositional changes, e.g. halide anion exchange. Such dynamic behavior has ultimately made understanding several important structure-property relationships ambiguous and obstructed a clear path toward commercialization due to inherent phase instability.

Our aim in this Account is to highlight the fundamental aspects of metal halide perovskites that dictate a stable crystal structure and enable efficient anion exchange, through the lens of thermodynamic preference and phase formation energies. Taking the all-inorganic $\mathrm{CsPbI}_{3-\mathrm{x}} \mathrm{Br}_{\mathrm{x}}$ system as a suitable case study, we focus on several ways in which its thermodynamically unstable perovskite structure can be maintained at room temperature and elucidate the restructuring pathways taken during destabilization. In addition, we will discuss the origin and mechanisms of post-synthetic anion exchange in $\mathrm{CsPbX}_{3}(\mathrm{X}=\mathrm{I}, \mathrm{Br}, \mathrm{Cl})$ perovskites, with emphasis made toward direct visualization using in situ optical micro-spectroscopy and arriving at quantitative results. For several notable features of halide perovskites dealt with in this Account - e.g. strain-stabilization, non-perovskite phase restructuring pathway and lattice anion diffusion - we attempt to rationalize them using stateof-the-art materials modelling techniques.

It is within this spirit that we not only modify a broad range of properties existing within metal halide perovskites, but regulate them, for enhanced material functionality. For example, controlling partial phase changes and local replacement of halide composition in $\mathrm{CsBX}_{3}(\mathrm{~B}=\mathrm{Pb}, \mathrm{Sn}$ and $\mathrm{X}=\mathrm{I}, \mathrm{Br}$, $\mathrm{Cl}$ ) nanowires can facilitate the formation of optoelectronic heterojunctions, due to the abrupt change 
in local crystal structure and the correlated transition in optoelectronic properties. From this combined perspective, metal halide perovskites appear as highly dynamic systems, whereby structural and compositional modifications have large a impact on the underlying phase stability and optoelectronic properties. Thus, we highlight several scientific aspects important to the fundamental understanding of metal halide perovskites, ranging from the underlying mechanism and kinetics through which phase destabilization and anion exchange take place, to tuning the thermodynamic energy landscape using external stimuli. We anticipate that providing a clear perspective for these topics will help deepen our knowledge of the nature of ionic semiconductors and provide the stimulus required to build new research directions toward utilizing halide perovskites within versatile optoelectronic devices.

\section{INTRODUCTION}

Following key discoveries in $2009^{1}$ and $2012^{2,3}$, the revival of organic-inorganic metal halide perovskite semiconductors has launched a new era of low-cost and high-performance photonics research $^{4}$. Their rise to prominence has been greatly influenced by their unique ability to combine facile solution processing methods with high-quality optoelectronic properties ${ }^{5}$; from tunable, efficient light absorption and emission at visible wavelengths, to long carrier lifetimes and diffusion lengths. Consequently, metal halide perovskites have found potential within a broad range of optoelectronic applications, most notably cheap and efficient solar cells ${ }^{6}$. This rapid progress has capitalized on over 20 years of research into related dye-sensitized and organic solar cells, and goes far beyond what was previously expected for devices based on solution-processed thin films ${ }^{7}$. While the desirable optoelectronic properties of halide perovskites have both driven and guided interesting research, their poor stability under typical operating conditions has played the role of its Achilles heel. This sentiment was recently highlighted by Yang and You ${ }^{8}$, where they urge the field to "Make Perovskite Solar Cells Stable".

In this Account, we will examine the topic of polymorphism and anion exchange (i.e. partial replacement of halide components) within halide perovskites research and develop a conceptual framework for understanding what triggers phase destabilisation at room temperature (RT). Focusing 
on all-inorganic halide perovskites, we summarize several different research lines we have taken to explore, understand, and regulate the phase energetics and material compositions, via anion exchange. A central theme of the research is a reliance on in situ characterisation and visualisation of phase transition phenomena, to correlate important (photo)physical aspects to the phase diagrams. For instance, in situ synchrotron-based X-ray diffraction has helped us elucidate the microstructural differences of strain-stabilized thin films, while in situ optical methods have helped reveal the underlying dynamics and mechanisms of anion exchange processes. Thus, precise control of perovskite phase and composition in nanocrystals, and the important optoelectronic properties, is shown possible. In terms of materials engineering for optoelectronic applications, we end the Account by highlighting the development of functional perovskite nanomaterials with highly tunable properties.

\section{THE METAL HALIDE PEROVSKITE STRUCTURE AND STABILITY}

Perovskites are materials that crystallize in the same structure as calcium titanate (CaTiO3), described by general formula $\mathrm{ABX}_{3}$ (Figure $\left.1 \mathrm{~A}\right)$. Here, $\mathrm{A}$ is cation, $\mathrm{B}$ is a divalent metal $(\mathrm{Pb}, \mathrm{Sn}, \mathrm{Ge})$, and $\mathrm{X}$ is a halide anion ( $\mathrm{I}, \mathrm{Br}$, or $\mathrm{Cl}$ ), with a lattice formed through a network of dynamic cornersharing octahedra, $\left[\mathrm{BX}_{6}\right]^{4-}$. For organic-inorganic lead halide perovskites, size restrictions within the ionic lattice necessitates the use of small A-site organic cations, e.g., methylammonium (MA: $\mathrm{CH}_{3} \mathrm{NH}_{3}{ }^{+}$) or formamidinium $\left(\mathrm{FA}: \mathrm{H}_{2} \mathrm{NCHNH}_{2}{ }^{+}\right.$). A central problem in the use of hygroscopic and volatile organic cations is their tendency to promote degradation pathways ${ }^{9-11}$, representing a major obstacle toward long-term stability. Alternatively, switching to all-inorganic caesium lead halide $\left(\mathrm{CsPbX}_{3}\right)$ perovskites allows for the coupling of high optoelectronic performance (e.g. solar cell photoconversion efficiencies above $18 \%^{12}$ ) with a huge improvement to the environmental stability ${ }^{13-}$ 15. The high-temperature (HT) black phase of $\mathrm{CsPbI}_{3}$-based perovskites ${ }^{16}$ has garnered the most attention due to its relatively narrow bandgap $(1.73 \mathrm{eV})$ and sensitive response to the solar spectrum. 


\section{A}

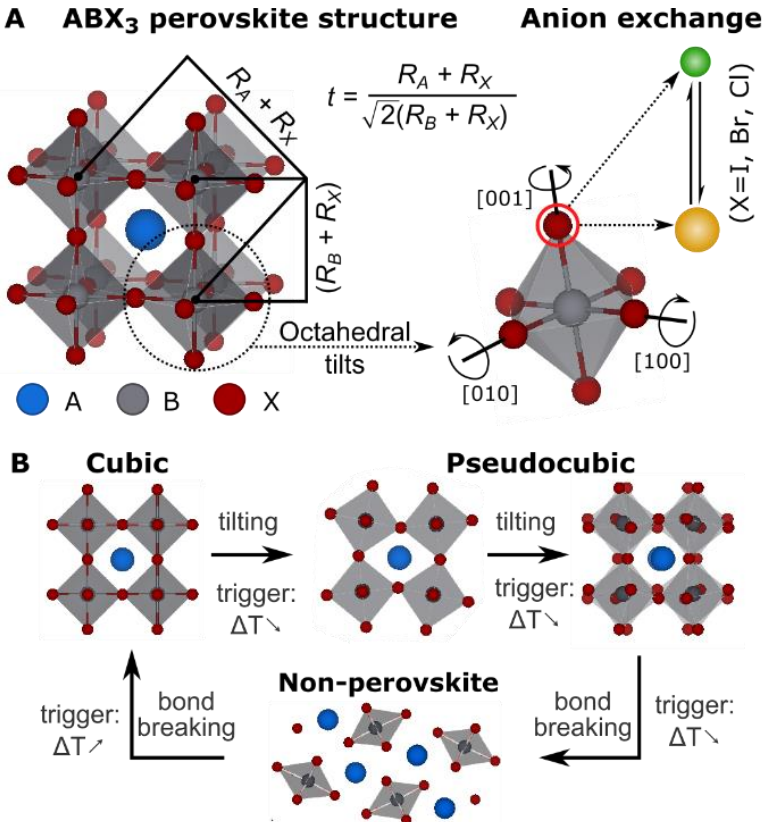

C
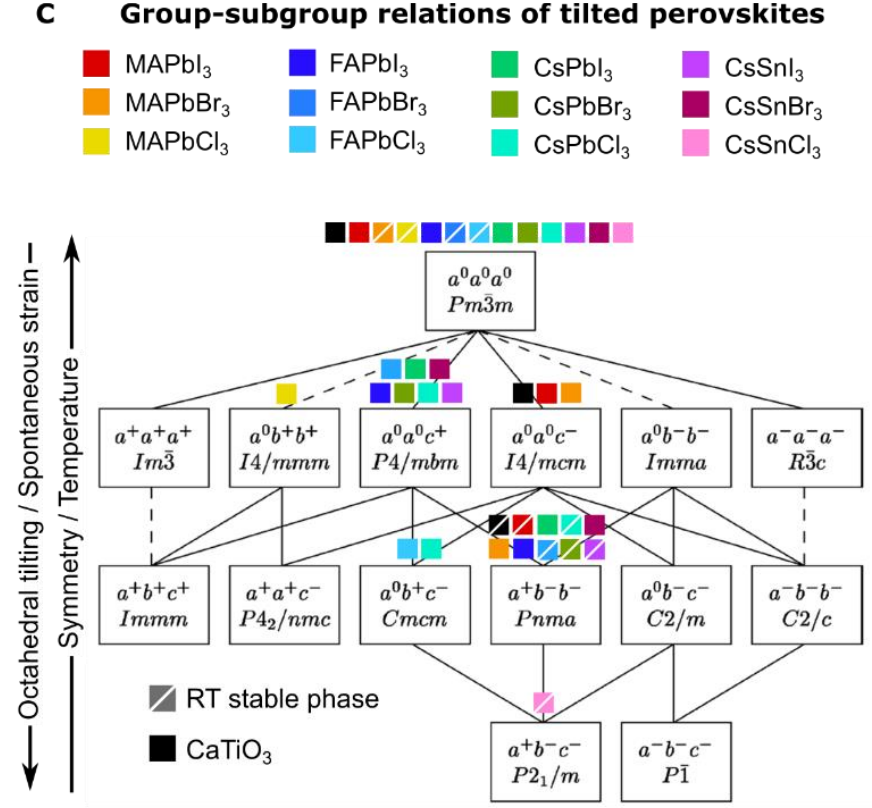

D Thermal phase relations

$\mathbf{E}$

Modified $\mathrm{CsPbI}_{3}$ energy diagrams
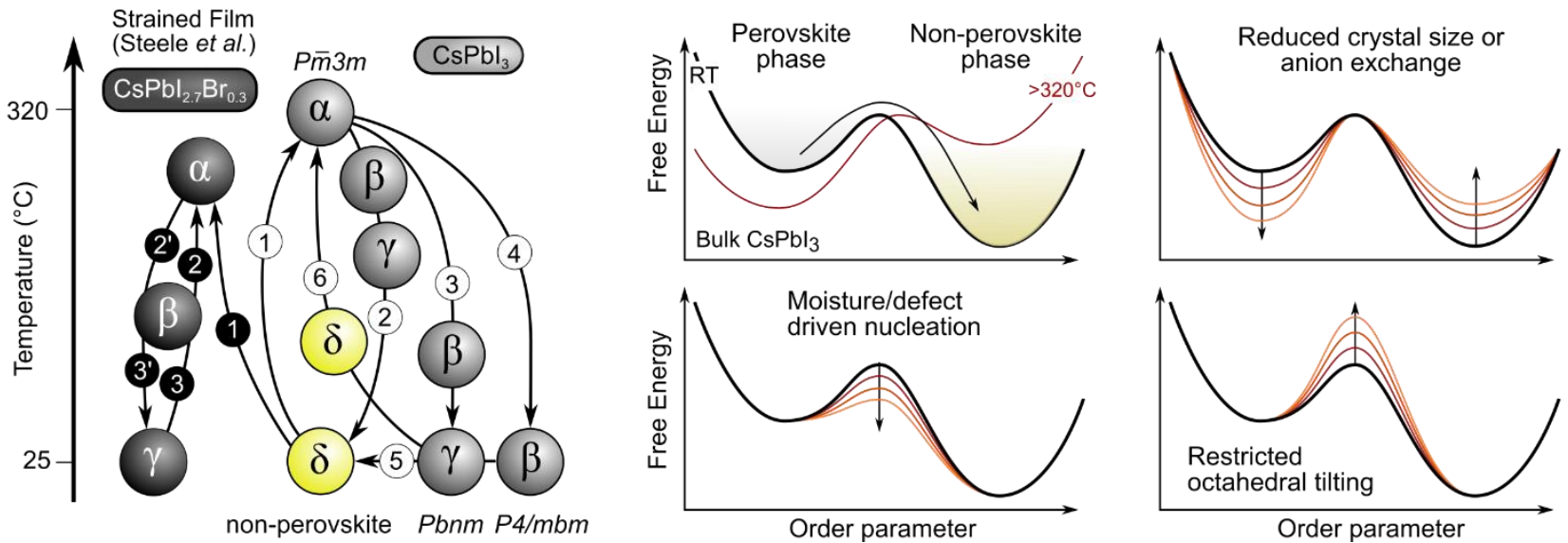

Figure 1. (A) The archetypal cubic crystal structure $(t=1)$ and anion exchange concept for $\mathrm{ABX}_{3}$ perovskites. When $t$ deviates from unity, octahedral tilts are introduced to accommodate a pseudocubic distorted perovskite structure. (B) Illustration of reversible phase transitions in polymorphic perovskites, transitioning though pseudocubic structures via octahedral tilting and into a nonperovskite structure via bond breaking. (C) Overview of reported group-subgroup symmetries of tilted halide perovskites $\mathrm{MAPbX}_{3}\left(\mathrm{X}=\mathrm{I}^{17}, \mathrm{Br}^{18}\right.$, and $\left.\mathrm{Cl}^{19}\right), \mathrm{FAPbX}_{3}\left(\mathrm{X}=\mathrm{I}^{20}, \mathrm{Br}^{21}\right.$, and $\left.\mathrm{Cl}^{22}\right), \mathrm{CsPbX}_{3}$ $\left(\mathrm{X}=\mathrm{I}^{23}, \mathrm{Br}^{24}\right.$, and $\left.\mathrm{Cl}^{25}\right)$, and $\mathrm{CsSnX}_{3}\left(\mathrm{X}=\mathrm{I}^{26}, \mathrm{Br}^{27}\right.$ and $\left.\mathrm{Cl}^{28}\right)$, with comparison to the archetypal $\mathrm{CaTiO}_{3}$ perovskite system ${ }^{29}$. The tilts are identified using Glazer's ${ }^{30}$ notation and the dashed lines correspond to first-order transitions. Other subgroups have been reported under atypical environments and are not included for simplicity. Adapted from Howard and Stokes ${ }^{31}$. (D) Overview of the thermal phase relations of pure $\mathrm{CsPbI}_{3}$ and the different paths which can be taken, depending on sample preparation and heating/cooling ramps. Adapted from Steele ${ }^{32}$. (E) Illustrative energy diagrams of bulk $\mathrm{CsPbI}_{3}$ (thick black lines) with comparisons to common modifications of the energy scheme. 


\subsection{Polymorphism}

Perovskites universally exhibit rich structural diversity, a property derived from the potential for the dynamic octahedra to tilt within its corner-sharing network (Figure $1 \mathrm{~A})$. The $\mathrm{ABX}_{3}$ perovskite structure is geometrically determined by the ionic radii of its constituent elements (i.e. $R_{A}, R_{B}$, and $\left.\mathrm{R}_{\mathrm{X}}\right)$; the ionic radii of common constituents are $\mathrm{A}=\mathrm{Cs}^{+}(1.88 \AA)^{33}, \mathrm{MA}^{+}(2.17 \AA)^{34}$ and $\mathrm{FA}^{+}(2.53 \AA){ }^{34}$; $\mathrm{B}=\mathrm{Pb}^{2+}(1.19 \AA)^{33}$ and $\mathrm{Sn}^{2+}(1.18 \AA)^{35} ; \mathrm{X}=\mathrm{Cl}^{-}(1.81 \AA)^{33}, \mathrm{Br}^{-}(1.96 \AA)^{33}$ and $\mathrm{I}^{-}(2.20 \AA)^{33}$. For a given temperature, $\mathrm{T}$, the relative bonding lengths can be used to calculate the Goldschmidt's tolerance factor (in the traditional formalism ${ }^{36}$ ).

$$
t=\frac{R_{A}+R_{X}}{\sqrt{2}\left(R_{B}+R_{X}\right)} .
$$

The concept of the Goldschmidt's tolerance factor ${ }^{37}$ (Figure 1A) is nearly a century old and has been widely used to gauge the phase stability, whereby a perovskite crystal is expected to form in the approximate range $0.8<t<1.1^{38}$. Thermal expansion will impose a continuous change in the unit cell volume by varying the internal bond lengths at different rates. For any given composition, this thermal response leads to the limited temperature ranges for which a stable perovskite phase may form. For instance, the characteristic low-temperature (LT: non-perovskite, yellow in color) to hightemperature (HT: perovskite, black in color) phase transition temperatures of $\mathrm{FAPbI}_{3}$ and $\mathrm{CsPbI}_{3}$ perovskites are well-above RT; $>125{ }^{\circ} \mathrm{C}$ for $\mathrm{FAPbI}_{3}{ }^{39}$ and $>315{ }^{\circ} \mathrm{C} \mathrm{CsPbI}{ }^{32}$. In contrast, due to its suitable molecular size, $\mathrm{MAPbI}_{3}$ is generally stable at $\mathrm{RT}^{40}$. The relatively high transition temperatures of $\mathrm{FAPbI}_{3}$ and $\mathrm{CsPbI}_{3}$ is what makes the phase stabilisation of the optoelectronically active black an important ongoing challenge within the field.

\subsection{Octahedral tilting}

Octahedral tilting is the source of both structural variation and destabilisation of the perovskite phase. Figure 1B illustrates how octahedral tilting prevents a corner-sharing network from collapsing while the temperature-dependent ${ }^{41}$ tolerance factor is shifted further away from unity, until bond bending is sufficient to break them and reform a non-perovskite (i.e. either side- or face-sharing octahedra). The restructuring of a parent cubic system into its lower symmetry sub-groups, and ultimately into a non-perovskite, is a fundamental feature in halide perovskites. Relative to an 
undistorted cubic perovskite $(\mathrm{Pm}-3 \mathrm{~m})$, Figure $1 \mathrm{C}$ overviews the 15 different space groups of tilted perovskite structures predicted using group theory by Howard and Stokes ${ }^{31}$. Glazer's notation ${ }^{30}$ classifies octahedral distortions which translate through the crystal ${ }^{42}$ during thermal expansion. Tilt identification can be understood using the general case depicted in Figure 1B, where subsequent layers being stacked along the tilt axis are tilted in-phase (+) and then antiphase (-), corresponding to the first and second cooling-induced tilts. Certain phase transition sequences are paralleled across different metal halide compositions within Figure 1C, highlighting the inherent tendency for perovskites to exhibit analogous phase behaviour. For instance, like the prototypical $\mathrm{CaTiO}_{3}$ perovskite, technologically important strontium zirconate $\left(\mathrm{SrZrO}_{3}\right)$ also exhibit the phase sequence upon heating $P n m a \rightarrow I 4 / m c m \rightarrow P m-3 m$, albeit at temperatures well above $1000{ }^{\circ} \mathrm{C}^{43}$.

\subsection{Anion exchange}

The flexible lattice and abundant halide vacancies in all-inorganic $\mathrm{CsBX}_{3}$ perovskites enable facile switching of the halide sites post synthesis (Figure 1A). Anion exchange is achieved by supplying the original $\mathrm{ABX}_{3}$ composition (i.e. $\mathrm{CsPbBr}_{3}$ ) with an abundant source of the precursor of another anion species (i.e. I-, Cl-) in solution or vapor phase, at elevated or room temperature. The large halide concentration gradient between the exterior and interior of the original structure drives the diffusion of guest ions into the parent lattice, to realize partial or even complete replacement of the original anions. As the crystal structures and bandgaps are dominantly dependent on the anion composition in lead halide perovskites, anion exchange thus provides a rich dimension for structural, physical, and optical diversity. For instance, alloying a portion of the iodine with relatively smaller bromine atoms in $\mathrm{CsPbI}_{3}$ has been shown effective at increasing $t$ closer to 1 at $\mathrm{RT}$, stabilizing the black phase ${ }^{44}$. Anion exchange can also shift the photoluminescence (PL) emission across the entire visible light spectrum $^{45}$, thus offering an effective post-synthetic transformation strategy for generating complex semiconductor heterostructures that are not accessible by direct synthesis methods. With anion exchange being demonstrated versatile for modifying the structures and properties in halide perovskite crystals, our research has sought to gain deep insights into the exchange mechanisms taking place. 


\subsection{Thermal phase relations of all-inorganic CsPbI3: a perfectly unstable case study}

The relatively small size of $\mathrm{Cs}^{+}$imposes a low $t$ value ( 0.85) for $\mathrm{CsPbI}_{3}$ at $\mathrm{RT}$, making the formation of stable perovskite materials and devices challenging. Figure 1D illustrates the thermal phase relations of the different $\mathrm{CsPbI}_{3}$, portraying its $\mathrm{HT}-\mathrm{CsPbI}$ black phases (so-called due to their comparable physical properties); cubic $(\alpha)$, tetragonal $(\beta)$ and orthorhombic $(\gamma)$. Upon cooling, the $\alpha-$ phase can pass through a variety of different restructuring paths, depending on conditions, The thermodynamically preferred cooling path (path 2$)^{23}$, though can remain in a HT-phase at RT (paths 3 and 4) if the driving force to transform into the low-temperature (LT) CsPbI3 phase is inhibited (path 5). The unstable phase energetics of bulk RT HT-CsPbI 3 is illustrated in Figure 1E. For instance, reheating kinetically trapped $\mathrm{RT}$ black $\mathrm{CsPbI}_{3}$ crystal (accessible through thermal quenching) to a temperatures of $60-100{ }^{\circ} \mathrm{C}$ is sufficient to destabilize $i^{46-48}$ (path 6). A central theme within our work has been to understand the transition mechanisms within the phase diagram outlined in Figure $1 \mathrm{D}$ and find ways to beneficially regulate it.

\section{EXTERNAL TRIGGERS AND THE MODIFIED ENERGY LANDSCAPE}

It is instructive to consider what happens to the phase diagram of $\mathrm{CsPbI}_{3}$ within the framework of its modified energy schemes (Figure 1E). To stabilize the black phase at RT, one can either shift the relative phase formation energies or increase the energy barrier, inhibiting the transition. Conversely, external triggers like structural defects and certain surface interactions which catalyse destabilisation should be avoided. Thus, beyond compositional changes, creating functional $\mathrm{CsPbI}_{3}$ perovskites unavoidably means diverging from a bulk-like state.

By reducing the crystal dimensionality a RT-stable $\mathrm{HT}-\mathrm{CsPbI} 3$ perovskite structure can be created via two separate influences. First, Luther et $\mathrm{al}^{49}$ demonstrated that an inverse dependence of the tolerance factor with size shifts $t$ closer to unity. Second, nanocrystals will experience a significantly modified surface energy contribution. Zhao et al. ${ }^{50}$ explored the relationship between the $\mathrm{CsPbI}_{3}$ crystallite size and the perovskite phase stability, highlighting a large disparity in the surface energy between the HT-phase $\left(0.13 \mathrm{~J} / \mathrm{m}^{2}\right)$ and LT-phase $\left(2.57 \mathrm{~J} / \mathrm{m}^{2}\right)$, whereby a reversal in the relative Gibbs free energies is expected for crystals smaller than roughly a $100 \mathrm{~nm}$-cubic. 
Surface functionalization ${ }^{51}$ (often involving organic ligands) is another widely adopted stabilization strategy, which ionically binds the crystal surface and imposes tilting restrictions. Crystal surfaces in general are known to house the tail ends of structure-property distributions, including bonding angles, and often facilitate nucleation sites for phase transitions. Thus, restricting the surface tilts can increases the transition energy barrier, inhibiting normal phase transition kinetics (Figure 1E).

\subsection{Straining to be black}
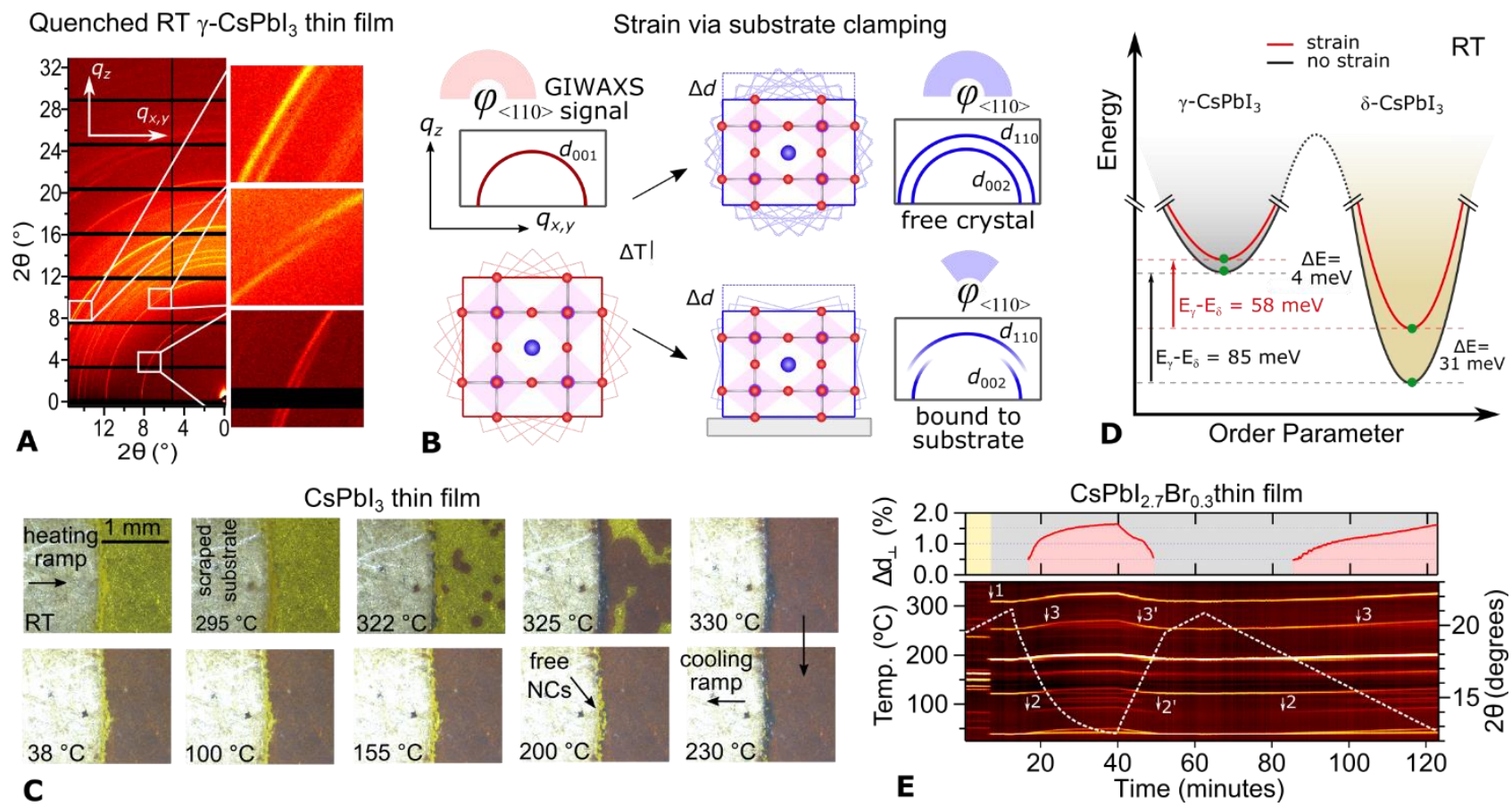

Figure 2. (A) GIWAXS image ( $\lambda=0.95774 \AA$ ) acquired from a quenched $\mathrm{RT} \mathrm{CsPbI}_{3}$ thin film, with expansions over selected diffraction peaks split in the in- and out-of-plane directions. (B) Schematic illustration of diffraction ring splitting in the GIWAXS signal, whereby a perovskite crystal forms a heterojunction with the substrate at high temperature and undergoes tensile strain and texture formation upon cooling. (C) Optical images of a partially scrapped $\mathrm{CsPbI}_{3}$ thin film surface (free NCs) recorded under $\mathrm{N}_{2}$ at different temperatures during a quenching profile. (D) Ab initio energy diagram indicating the relative stability (at $0 \mathrm{~K}$ ) of the $\mathrm{HT}-\mathrm{CsPbI}_{3}$ and $\mathrm{LT}-\mathrm{CsPbI}$ phases with and without in-plane biaxial strain. Note that the relative saddle point depth is undefined. (E) 
GIWAXS $t$ - $T$ profile of $\mathrm{CsPb}_{2.7} \mathrm{Br}_{0.3}$ thin film through a high-temperature LT-to-HT phase transition, followed by thermal cycling. Adapted from Steele ${ }^{32}$.

Introducing strain is an established path toward structural and compositional diversity in metastable semiconductors crystals ${ }^{52}$, because strain will inherently shift the system away from normal equilibrium conditions. For instance, when oxide perovskite thin films form heterojunctions with the underlying substrate and become clamped, the film must track any mechanical changes undertaken by the substrate, which can dramatically alter the free energy landscape of competing ferroelectric phases ${ }^{53,54}$. Recently, Zhao et al. ${ }^{55}$ demonstrated that high temperature annealing of organic-inorganic halide perovskite thin films formed strong interfaces with the substrate, regardless of the composition of the perovskite or substrate. Using synchrotron-based grazing incident wideangle X-ray scattering (GIWAXS) we examined the role that substrate clamping has on shifting the phase energetics in $\mathrm{CsPbI}_{3}$ perovskite thin films. Resolving the 2D micro-structure (i.e. in- and outof-plane) of a thermally quenched $\mathrm{HT}-\mathrm{CsPbI}_{3}$ thin film in Figure $2 \mathrm{~A}$ we found the crystal to be heavily strained by the substrate at RT. This occurs via two steps; (i) high temperature processing $\left(330{ }^{\circ} \mathrm{C}\right)$ both transitions the $\mathrm{CsPbI}_{3}$ film to the HT-phase and establishes a hetero-interface, as the soft perovskite crystal adapts to the substrate surface ${ }^{55}$, and (ii) cooling biaxially strains the clamped thin film (Figure $2 \mathrm{~B})$, which has a much larger thermal expansion coefficient $\left(\alpha_{\mathrm{T}} \sim 50 \times 10^{-6} \mathrm{~K}^{-1}\right)$ than the underlying substrate $\left(\alpha_{\mathrm{T}} \sim 4\right.$ to $\left.9 \times 10^{-6} \mathrm{~K}^{-1}\right)$.

Strain alters the energetics of the competing LT- and HT-phases ${ }^{16,53,56}$, an effect vividly captured by us using in situ optical microscopy in Figure 1C; the material still clamped to the substrate becomes trapped in the $\mathrm{HT}-\mathrm{CsPbI}_{3}$ state at RT, whereas the detached materials readily turn to LT$\mathrm{CsPb}_{3}$. The processes taking place during annealing underpin this observation of modified phase energetics (Figure 1D). First, the hetero-interface will increase the energy barrier height via restricted tilting, and cooling-induced strain will renormalize the phase formation energies (Figure 2D). To elucidate the strain-induced shift in phase energetics, we calculated its response using DFT over 12 different planar strain directions. The averaged energy values are shown in Figure 2D confirming that the unstrained LT-phase is strongly favored over its HT-phase counterpart. Notably, however, biaxial strain imposes different energy penalties for the two phases and introduces a relative stabilization of the strained black perovskite structure. We further experimentally confirmed this response on other 
substrate surfaces and found that films grown with a thickness below a micron also exhibited similar strain profiles.

Evaluating the high temperature processing of a $\mathrm{CsPbI}_{2.7} \mathrm{Br}_{0.3}$ thin film using in situ GIWAXS through multiple thermal cycles (Figure 2E), we found that the normally preferred LT-phase is replaced with a stable HT-phase after establishing a stained interface during the first heating ramp. Such thermodynamic behaviour is highlighted in Figure 1E and provides the kind of thermal stability desirable for NIR HT-CsPbI 3 -based optoelectronic devices. Using this same thin film composition, we demonstrated a functioning LED device based on a strain-stabilized $\mathrm{CsPbI}_{2.7} \mathrm{Br}_{0.3}$ active layer.

\subsection{Moisture-induced shifts to the $\mathrm{CsPbI}_{3}$ phase diagram}
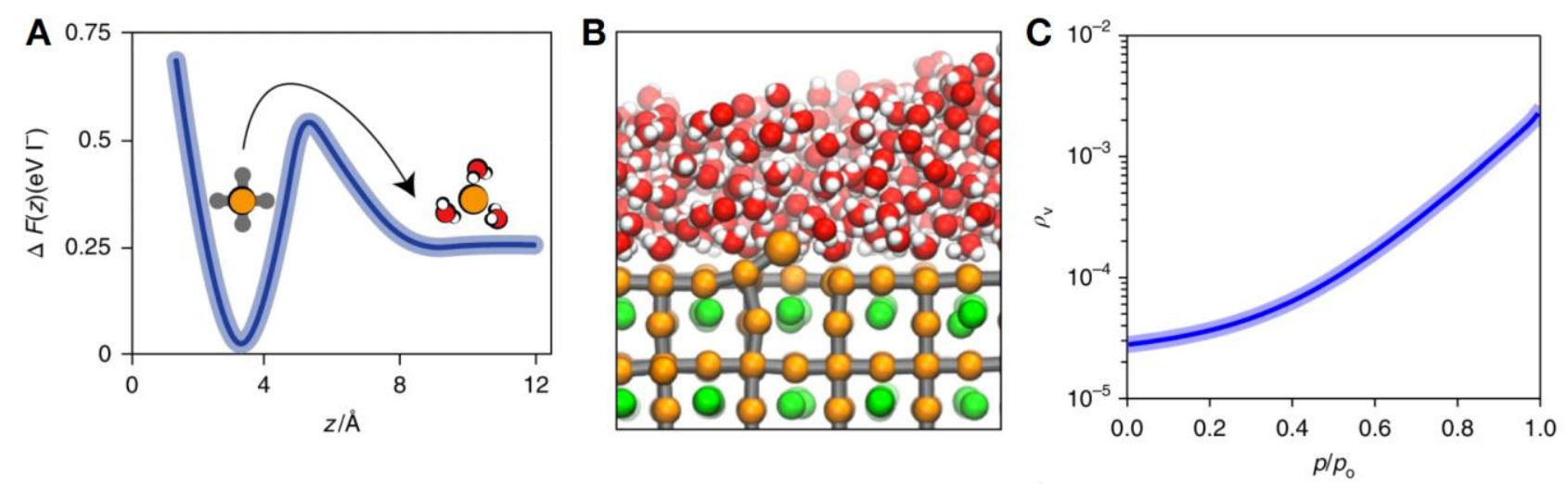

Figure 3. (A) Reversible work, $\Delta \mathrm{F}(\mathrm{z})$, required to transfer an $\mathrm{I}^{-}$atom from the solid perovskite to the thin adsorbed water layer, where direction $\mathrm{z}$ perpendicular to the interface. (B) MD simulation snapshot near the top of the energy barrier in (A), showing the lead halide sub-lattice (grey and orange), $\mathrm{Cs}^{+}$cations (green), and water molecules (red and white). (C) Calculated iodide vacancy concentration, $\rho_{v}$, per unit cell as a function of relative humidity, $p / p_{o}$. Adapted from ${ }^{57}$.

The ionic lattice of metal halide perovskites is water-soluble, leading to intrinsically poor stability under ambient operating conditions. When exposed to moisture or other polar molecules, the crystal surface can disassociate and degrade, stifling performance. Further, the metastable black HTphase of $\mathrm{CsPbI}_{3}$ has been widely reported to be sensitive to moisture ${ }^{46}$. For example, our strained black $\mathrm{CsPbI}_{3}$ thin films were shown to decay and turn yellow ${ }^{32}$ within just several minutes of being exposed to an ambient atmosphere (27\% RH). 
We recently examined this problem in detail and tried to understand the underlying mechanisms of so-called moisture attack ${ }^{57}$ and how it accelerates the HT- to LT-phase transition. Unlike the chemical decomposition in hybrid perovskite $\mathrm{MAPbI}_{3}, \mathrm{H}_{2} \mathrm{O}$ molecules only adsorb on $\mathrm{CsPbI}_{3}$ surface and partially dissolve the halide anions. As a result, the increased halide vacancy concentration can lower the kinetic barrier and catalyse yellow phase formation, as outlined in Figure 3. Molecular dynamics (MD) simulations (Figure 3A and B) show an enhancement of halide vacancy formation at the water-perovskite interface due to the large solvation enthalpy of halide ions and low vacancy formation energy in the perovskite lattice. Consequently, the equilibrium halide vacancy concentration at the crystal surface is expected to rise as a function of increases relative humidity, as depicted in Figure 3C. Through increased adsorption of $\mathrm{H}_{2} \mathrm{O}$ molecules, the equilibrium halide vacancy concentration can be enhanced up to five orders of magnitude compared to the bulk interior. Using ambient pressure X-ray photoemission spectroscopy (AP-XPS), we showed that water molecule catalysts are adsorbed only onto the surface without diffusing inside the crystal. Further, halide vacancies reduce the surface tension between the two phases and facilitate faster transition rates, a feature consistent with the experimental observations of faster transition times when the relative humidity is increased from $20 \%$ to $80 \%$. Like water, we found other polar solvents such as methanol or ethanol can also trigger the phase transition, although their catalytic performances are not as efficient as water due to their weaker polarity.

\subsection{Transitions between the LT and HT phases}

The distinct difference in crystal structure between the perovskite and non-perovskite phases ultimately equates to fundamental differences in their photophysical properties, enabling the formation of phase-change optical devices. Such devices developed by us will be highlighted later. In order to understand the phase transition mechanisms and kinetics, we have used halide perovskite nanowires (NWs) as a model platform for investigating the rich solid-solid phase transition dynamics at a microscopic scale. We directly synthesized non-perovskite $\delta$ - $\mathrm{CsPbI}_{3} \mathrm{NW}$ structures via a lowtemperature substrate-guided method utilizing its anisotropic internal crystal structure. Through heating $\left(\sim 320^{\circ} \mathrm{C}\right)$ and a rapid thermal quench of the as-grown $\delta$ - $\mathrm{CsPbI}_{3} \mathrm{NWs}$, they are transformed into a RT $\gamma$-phase. Additionally, the phase transition temperature can be reduced to $\sim 150{ }^{\circ} \mathrm{C}$ via alloying of $\mathrm{CsPbI}_{3}$ with $\mathrm{Br}$, to form $\mathrm{CsPbIBr}_{2}$. Once transformed into the perovskite phase, the NWs 
exhibited enhanced luminescence efficiency and a relatively narrow bandgap, enabling direct observation of the non-perovskite-to-perovskite phase transition dynamics using advanced nanoimaging techniques.
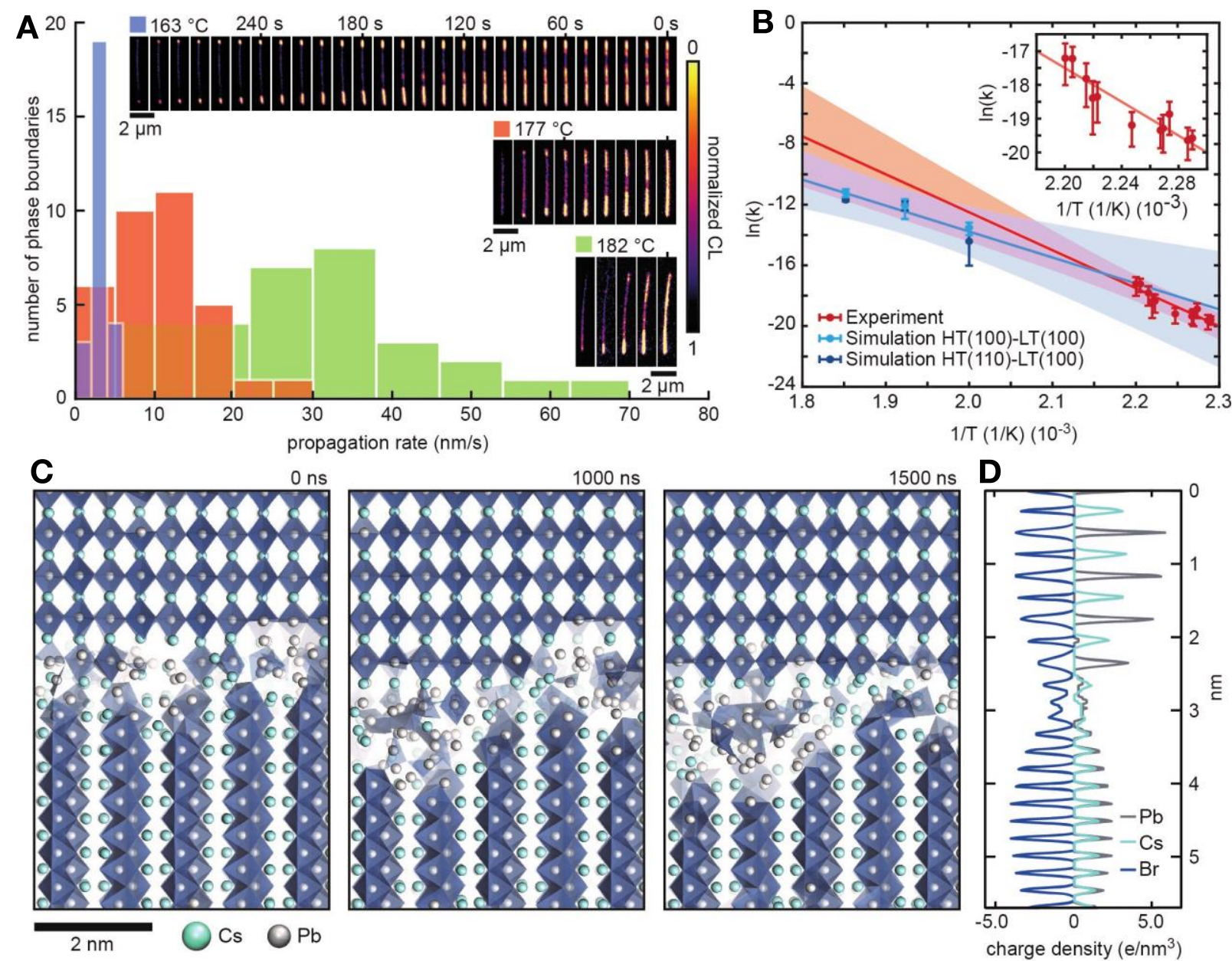

Figure 4. (A) Histograms of phase propagation rate of $\mathrm{CsPbBr} \mathrm{I}_{2} \mathrm{NWs}$ heated to three different temperatures. The insets show characteristic NWs corresponding to different times of the phase conversion. (B) Arrhenius plot of experimental and MD simulation data; the solid red line is the linear fit to the experimental data $(210 \pm 60 \mathrm{~kJ} / \mathrm{mol})$, and the solid blue line is the linear fit to the simulation data $(140 \pm 80 \mathrm{~kJ} / \mathrm{mol})$. The inset is a detailed plot of the experimental data. (C) Snapshots from the MD simulation of phase propagation as a function of time at $267^{\circ} \mathrm{C}$, showing the disordered interface between the LT and HT phases of $\mathrm{CsPbBr}_{3}$. Here we have parameterized a classical forcefield for $\mathrm{CsPbr}_{3}$ crystals, which is used as a proxy for the structurally similar mixed halide perovskite studied experimentally. (D) Charge density profiles from the MD simulation at $267^{\circ} \mathrm{C}$, obtained by projecting 
ion positions onto the direction of the wire axis. Data are averaged over a 50 ns time window. Reproduced from Bischak ${ }^{58}$.

Nanoscale cathodoluminescence (CL) microscopy provides high spatial resolution and excellent sensitivity by exciting optically emissive materials with a scanning electron beam. Recently we applied CL in situ while heating and imaged the phase transition process from $\mathrm{LT}-\mathrm{CsPbIBr} 2$ to HT-CsPbIBr 2 . Like LT-CsPbI 3 NWs, as-synthesized LT-CsPbIBr 2 NWs have a similar edge-sharing structure containing metal halide octahedral chains oriented along the major axis of the NW. As seen in Figure 4A, nucleation of the emissive HT-CsPbIBr 2 phase was typically observed at the NW ends, as indicated via bright CL emission and subsequent propagation along the wire. Through observing hundreds of $\mathrm{CsPlBr}_{2}$ NWs, we arrived at a statistical distribution for temperature-dependent (163 ${ }^{\circ} \mathrm{C}, 177{ }^{\circ} \mathrm{C}$, and $182{ }^{\circ} \mathrm{C}$ ) phase propagation rates (Figure $4 \mathrm{~A}$ ); the propagation rates are found to be $3.1 \pm 0.2 \mathrm{~nm} / \mathrm{s}, 11 \pm 1 \mathrm{~nm} / \mathrm{s}$, and $33 \pm 3 \mathrm{~nm} / \mathrm{s}$, respectively. In addition, we determined an activation energy of $210 \pm 60 \mathrm{~kJ} / \mathrm{mol}$ via Arrhenius fitting (Figure 4B), with the propagation rate as a function of temperature. A significant increase in entropy is expected to explain such a large energetic barrier, instead of the enthalpy change $(\sim 15 \mathrm{~kJ} / \mathrm{mol})$.

To understand the microscopic mechanism of phase propagation, we applied a classical force field to perform MD simulations in a simpler composition of $\mathrm{CsPbBr}_{3}$. Figure $4 \mathrm{C}$ shows the MD simulations with (100) $\mathrm{LT}-\mathrm{CsPbBr} 3$ attaching to (100) $\mathrm{HT}-\mathrm{CsPbBr}_{3}$ at a phase interface, as determined by ex-situ selective area electron diffraction (SAED) ${ }^{58}$. The simulations also suggest that the compensating increase in entropy promotes the formation of a structurally disordered amorphous interfacial layer, which helps to propagate the interphase boundary. The phenomena of phase propagation from our simulations agrees with the experimentally derived value of $140 \pm 80 \mathrm{~kJ} / \mathrm{mol}$ for the activation energy (Figure 4B). The growth of the $\mathrm{HT}-\mathrm{CsPbBr}_{3}$ structure is thus suggested propagates via ion diffusion across the amorphous phase interface (Figure 4C) instead of a martensitic transformation. The mobile halide anions in the perovskite lattice are likely to guide the formation of HT-phases, as the halide species are distributed in a long-range order across the amorphous interface (Figure 4D).

\subsection{Structure-property relationships in polymorphic crystals}

Phase transitions involving a corner-sharing tilts present a much lower energy barrier compared to transitions requiring bond-breaking to form a non-perovskite structure. During phase transitions, certain properties will change, often discontinuously, in response to the change of external 
conditions, such as temperature or pressure. For example, we showed that phase transitions in $\mathrm{CsPb}_{\mathrm{x}} \mathrm{Sn}_{1-\mathrm{x}} \mathrm{I}_{3} \mathrm{NWs}^{59}$ are accompanied by both a dramatically altered optical bandgap and electrical conductivity. This is what brings complexity to understanding the dynamical nature of such transitions in devices operating near RT, and connecting them to physical features which manifest at pre-phase transition boundaries. For a clear example, whether as-synthesized $\mathrm{CsPbBr}_{3}$ quantum dots form a truly cubic structure at RT has been hotly debated.

The radiation-sensitive nature of halide perovskites has hindered structural studies at the atomic scale using high-energy electron beams. We have overcome this obstacle by applying lowdose high-resolution transmission electron microscopy to image the pristine atomic structure of ultrathin $2 \mathrm{D} \mathrm{CsPbr}_{3}$ perovskites ${ }^{60}$. Both orthorhombic and cubic perovskite phases of $\mathrm{CsPbBr}_{3} \operatorname{are}$ found to coexist in a single particle, probably owing to the small energy difference between their phase transitions. Thus, near the phase transition boundaries discontinuities in the optoelectronic properties will exist. Beyond abrupt changes in the PL peak position during the phase transitions in $\mathrm{MAPbI}_{3}$, we have correlated electrical transport data to these phase transitions. Specifically, we performed longitudinal resistance $\left(R_{x x}\right)$ measurements under steady-state illumination as a function of temperature, with the structural transitions tracked using linear thermal expansion ${ }^{61}$. We found that upon cooling down to cryogenic temperatures the electronic resistance decreased by a factor of two. However, precisely at the $\mathrm{MAPbI}_{3}$ tetragonal-to-orthorhombic perovskite phase transition, $R_{x x}$ sharply rises by over an order of magnitude before dropping again, to continue its decreasing trend while cooling in the orthorhombic regime. Such insights highlight how important it is to understand the (pre-)phase transition kinetics, which dramatically alter properties central to device performance.

In low dimensional crystals, like Ruddlesden-Popper (RP) phases consisting of two-dimensional perovskite slabs interleaved with cations, thermal-induced modifications to both phase and optoelectronic properties become interesting due to the interplay between the alternating layers. For instance, synthesizing single crystal $(B A)_{2} \mathrm{Cs}_{n-1} \mathrm{~Pb}_{n} \mathrm{Br}_{3 n+1}$ with different layer numbers (n) we discovered ${ }^{62}$ that the tilted $\left[\mathrm{PbBr}_{6}\right]^{4-}$ octahedra in the organic-inorganic RP phase perovskites align along the [100] axis when the temperature increases from $100 \mathrm{~K}$ to $400 \mathrm{~K}$. Further increasing in temperature induces a first-order phase transition from the orthorhombic to tetragonal space group, and the organic chains disorder. The structural dynamics correlate with a sudden drop in the PL intensity and consequently with LED device degradation, indicating heat management and phase control are essential to maintaining high performance devices.

Beyond structural phase transitions, distinct electronic transitions (i.e. semiconductor to metal transition, high $\mathrm{T}_{\mathrm{c}}$ superconducting) are predicated theoretically in charge-ordered halide perovskites. 
To study such phenomena, we synthesized an In-based, charge-ordered $\left(\mathrm{In}^{+} / \mathrm{In}^{3+}\right)$ inorganic halide perovskite $\mathrm{Cs}_{2} \mathrm{In}(\mathrm{I}) \mathrm{In}(\mathrm{III}) \mathrm{Cl}_{6}$ single crystals via a solid-state reaction method ${ }^{63}$. Pressure-dependent optical absorbance spectroscopies further reveal the abrupt vanishing of the fundamental semiconductor bandgap, whereby increased absorption with a broadband metallic character is introduced. The experimental demonstration of pressure-induced semiconductor-to-metal phase transition was consistent with theoretical modeling, which may open a pathway toward the discovery and understanding of quantum phase transition in halide perovskites and inspire potential applications in quantum devices.

\section{CONTROLLING PHASE AND ANION EXCHANGE AT THE NANOSCALE}

Due to the high anion mobility and the rigidity of the cationic sublattice, anion exchange reactions preserve together the crystal structure, morphology and material quality of halide perovskites. As the halide composition directly governs the local optical properties, controlling the anion exchange reactions has proven useful in designing and forming semiconductor heterostructures which can be tracked readily by in situ PL microscopy. Such studies have yielded important kinetic information directly ${ }^{64,65}$ and, by developing different reaction procedures in solution and gas phases, we have quantitatively investigated the underlying physical mechanisms involved in the anion exchange process. In the end, precision of the anion exchange process has permitted us to realize novel optoelectronic heterojunctions. 

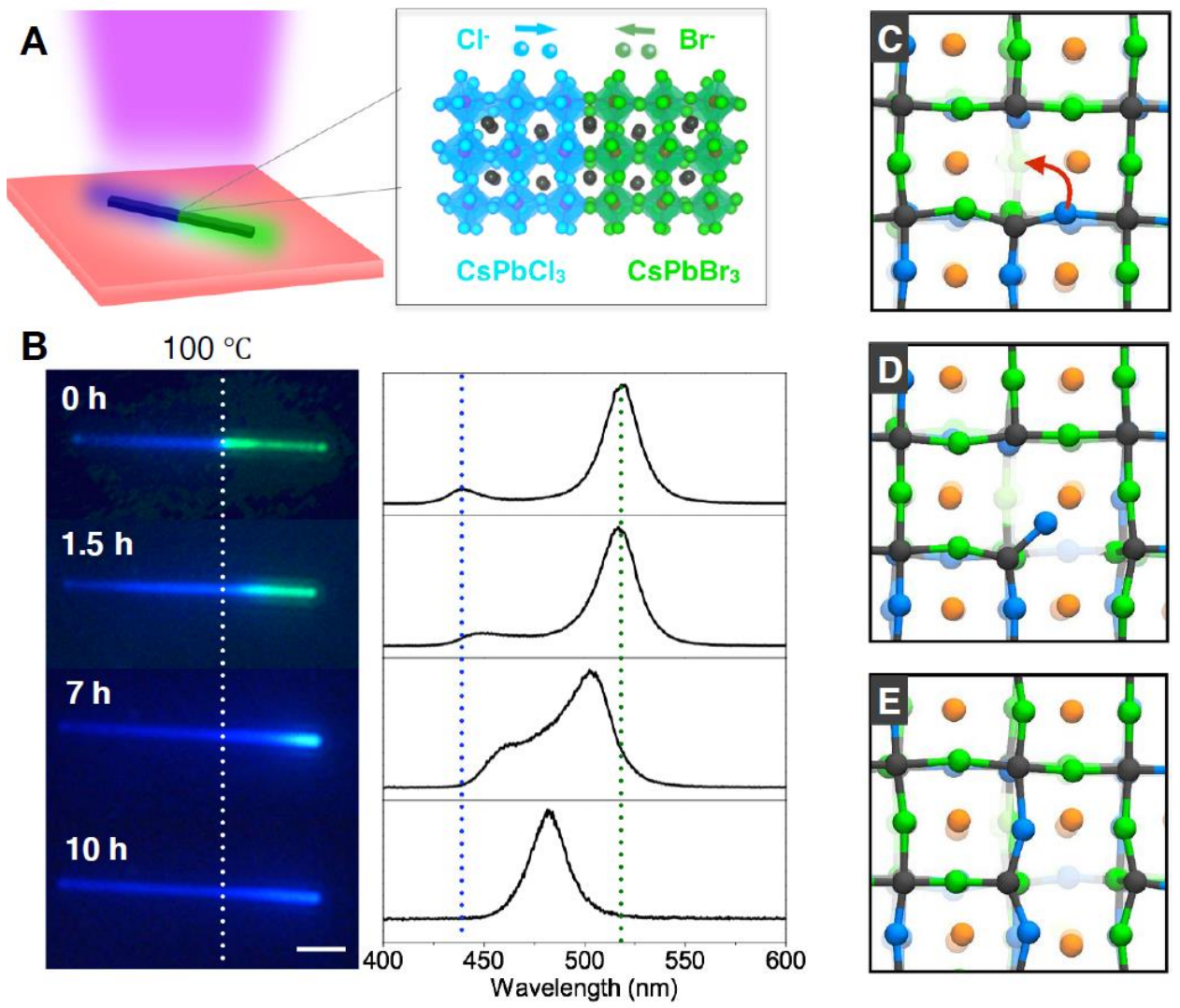

Figure 5. (A) Schematic illustration of wide-field PL measurement of the thermal-driven anion interdiffusion on a $\mathrm{CsPbCl}_{3}-\mathrm{CsPbBr}_{3}$ heterojunction NW. (B) PL images and corresponded emission spectra for the heterojunction NW when heated constantly at $100{ }^{\circ} \mathrm{C}$ for different snapshots in time. Here the heterointerface moves towards the Br-rich region while the PL spectrum changes accordingly, with the merging of two peaks into one. Scale bar: $2 \mu \mathrm{m}$. (C-E) Snapshots of the MD simulation of halide interdiffusion. A chloride anion swaps with a vacancy in an equal-molar mixture, in the initial position (C), on top of the energy barrier (D), and in the final position (E). Color code: $\mathrm{Cs}$ in orange, $\mathrm{Pb}$ in black, $\mathrm{Br}$ in green, and $\mathrm{Cl}$ in blue. The sticks represent the strong lead halide ionic bonds. Reproduced from Lai ${ }^{64}$.

\subsection{Mechanisms of anion exchange and regulating the perovskite phase}

Through temperature-dependent measurements of reaction rates, the activation energy values for both the $\mathrm{Br}^{-}$to $\mathrm{I}^{-}$and the $\mathrm{Br}^{-}$to $\mathrm{Cl}^{-}$exchanges were determined to be positive by Alivisatos et al., suggesting the anion exchange is a kinetic process, with either surface reaction- or diffusion-limited mechanisms ${ }^{66}$. The anions in perovskite structures are known to be highly mobile, so understanding the anion diffusion inside the solid-state lattice is essential to understanding of the anion exchange 
phenomena. Within this context, we studied joined $\mathrm{CsPbCl}_{3}-\mathrm{CsPbBr}_{3} \mathrm{NW}$ heterojunctions to monitor the interdiffusion of $\mathrm{Br}^{-}$and $\mathrm{Cl}^{-}$across the two segments, by thermally-triggering the exchange and visualizing the dynamics via wide-field PL imaging (Figure 5A). Heating the NW at a constant temperature $\left(100{ }^{\circ} \mathrm{C}\right)$ over different periods of time (Figure 5B), the PL peak evolution was tracked and both the Br-rich (green) and Cl-rich (blue) regions of the NW where spatially mapped. By analyzing the local $\mathrm{Br} /(\mathrm{Br}+\mathrm{Cl})$ ratio along the $\mathrm{NW}$ as a function of time the halide interdiffusivities were determined to be $10^{-13}-10^{-12} \mathrm{~cm}^{2} / \mathrm{s}$ at $75-125{ }^{\circ} \mathrm{C}$. Plots of the temperature-dependent interdiffusion coefficients further revealed the activation energies for the halide diffusions to be 0.28 $\mathrm{eV}$, by fitting with an Arrhenius equation.

Regarding the underlying origins of diffusion, our MD simulations suggest a halide vacancymediated mechanism (Figure 5C-E), given that the computed halide vacancy formation free energies are anticipated to be much smaller, leading to the high equilibrium vacancy concentrations that dominate the high interdiffusivities. As schematically illustrated in Figure 5C-E, the anions need to overcome the free energy barriers to diffuse by exchanging their positions with vacancies. It follows that intrinsic ion diffusion is facilitated by the soft lattice of halide perovskites, because of the low barriers for vacancy hopping revealed through MD simulations ${ }^{64}$.

By allowing vaporized n-Butylammonium iodide to react with a set of $\mathrm{CsPbBr}_{3}$ nanoplates (20 to $350 \mathrm{~nm}$-thick) simultaneously, Br- is systematically swapped for I- during our in situ PL probing and enables the anion exchange kinetics to be decoupled into two separate stages; a surface exchange reaction followed by subsurface ion diffusion. Microscopic confocal PL imaging of the nanoplates at different reaction stages shows a clear dependence of the anion exchange rates on both reaction time and plate thickness. Using a first-order description for the surface reaction and modelling the 1D vertical diffusion by Fick's second law, the iodide diffusion coefficient in the nanoplates was estimated to be on the order of $10^{-14} \mathrm{~cm}^{2} / \mathrm{s}$ at $\sim 180{ }^{\circ} \mathrm{C}$. Such diffusion-limited transformations ultimately led to the controlled formation of $\mathrm{CsPbX}_{3}$ heterostructures, with distinctly separate $\mathrm{Br}$-rich and I-rich regions ${ }^{65}$.

Anion exchange offers an appropriate route for accessing structures that are thermodynamically unstable or inaccessible via direct synthesis techniques. For example, the direct 
synthesis of $\mathrm{CsPbI}_{3} \mathrm{NWs}$ unavoidably results in a wide bandgap $(\sim 2.8 \mathrm{eV})$ yellow phase with poor optoelectronic performance ${ }^{67}$. Alternatively, starting from $\mathrm{CsPbr}_{3}$ which forms a stable perovskite near RT, exchanging the $\mathrm{Br}$ for I resulted in a mixed alloy or fully exchanged $\mathrm{CsPbI}_{3}$ materials, preserving the perovskite structure of the original host. Via exchange, a narrow, solar friendly bandgap as low as $1.76 \mathrm{eV}$ can be realized. Further, the colloidal $\mathrm{CsPbI}_{3} \mathrm{NWs}$ resulting from anion exchange retained high PL quantum yields of around 81\%, without any increase to defect densities, compared to the parent $\mathrm{CsPbBr}_{3}{ }^{68}$ systems.

The differing optical properties of the LT- and HT-phases expressed by all-inorganic $\mathrm{CsPbI}_{\mathrm{x}} \mathrm{Br}_{3-\mathrm{x}}$ materials offer an interesting opportunity to reversibly switch between them, for environmentally driven thermochromic application. Using heat and moisture as triggers, we developed $\mathrm{CsPbBr}_{2} \mathrm{I}$ thermochromic windows exhibiting varied optical transparency, depending on the environmental conditions (Figure 6A). At RT, the wide-bandgap (2.9eV), thermodynamically stable LT-phase is relatively transparent to visible light. Heating the window quickly transforms it to the HT-phase, changing its transparency from $81.7 \%$ to $35.4 \%$ and is a dark in appearance. Using this approach, both the transition temperature $\left(105\right.$ to $\left.320^{\circ} \mathrm{C}\right)$ and band gap $(1.7$ to $2.3 \mathrm{eV}$ for black phase), and thus transparency, can be tuned by varying the bromine to iodine ratio. Using a composition of $\mathrm{CsPbIBr}_{2}$, for example, solar cell made from such an absorber exhibited PCEs above $7 \%$ and maintained more than $85 \%$ of this efficiency after 40 consecutive phase transition cycles.

Using direct laser write (DLW; cw $458 \mathrm{~nm}$ ) as a local thermal trigger ${ }^{69}$, we also explored the possibility of microprocessing functional $\mathrm{HT}-\mathrm{FAPbI}_{3}$ perovskite elements embedded into relatively insulating LT-phase single crystals. Due to the poor thermal conductively of perovskite in general $^{70}$, rapid DLW allowed for controlled restructuring of optically-active $\alpha-\mathrm{FAPbI}_{3}$ microstructures with lateral dimensions nearing the diffraction limit $(<1 \mu \mathrm{m})$. Interestingly, unlike heated $\mathrm{FaPbI}_{3}$ which lasts just a few days in the HT-phase, DLW transformations were lasting months under ambient conditions, likely due to the stabilizing heterointerface established between the LT- and HT-phases. 


\subsection{Fabricating functional heterojunctions}
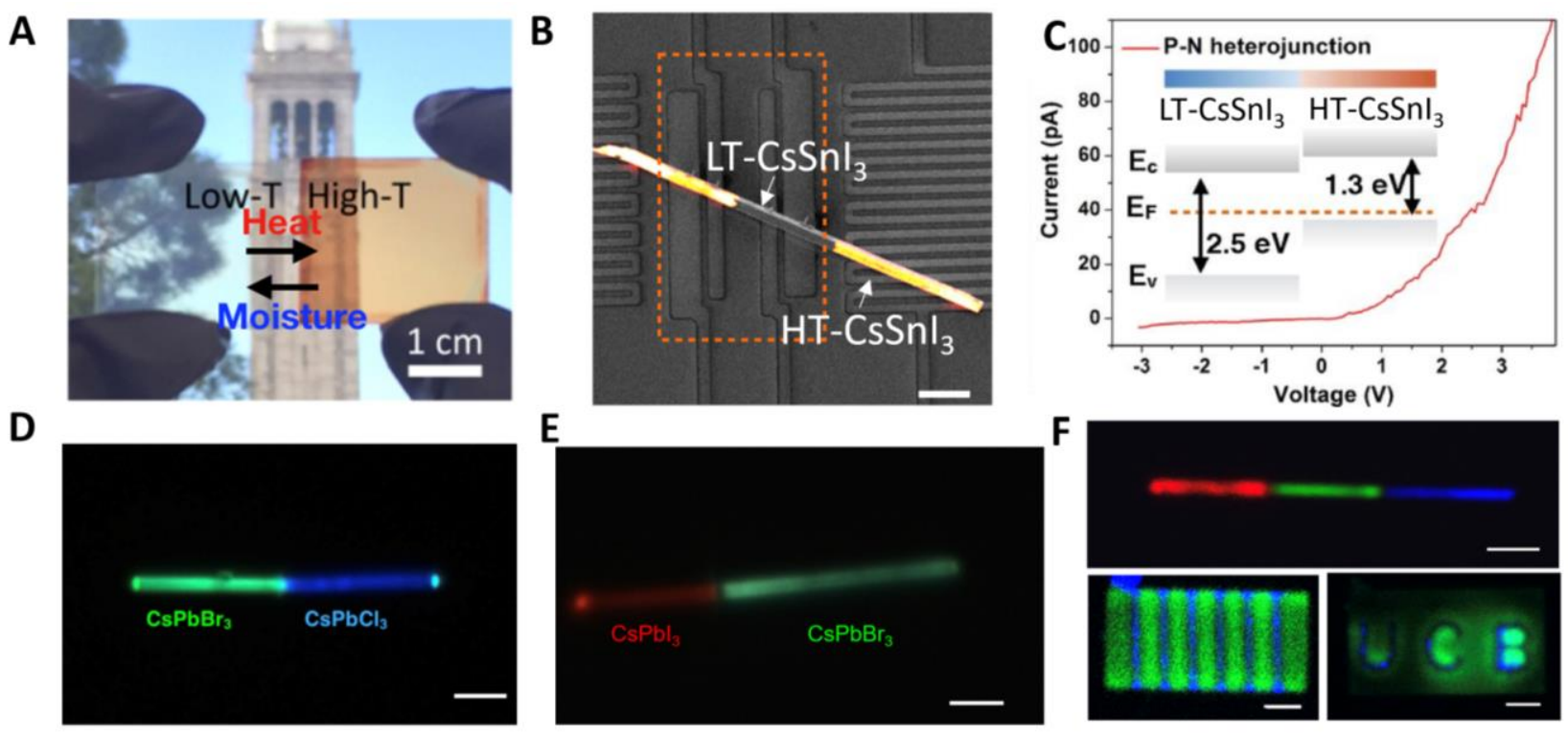

Figure 6. (A) Photograph of LT-phase and HT-phase thin films, an interconversion between the two made reversible via heating and exposure to moisture. Adapted from $\operatorname{Lin}^{57}$ (B) CL images of P-N heterojunction formed through a localized phase transition in a single $\mathrm{CsSnI}_{3} \mathrm{NW}$ (Scale bar: $5 \mu \mathrm{m}$.). (C) I-V characteristics of the P-N heterojunction shown in (B), measured using the four central electrodes. The Inset shows the relative energy band alignment of LT-CsSnI 3 and HT-CsSnI 3 under thermal equilibrium. Confocal PL mapping of (D) $\mathrm{CsPbBr}_{3}-\mathrm{CsPbCl}_{3}$, (E) $\mathrm{CsPbI}_{3}-\mathrm{CsPbBr}_{3} \mathrm{NWs}$ and (F, top) $\mathrm{CsPbI}_{3}-\mathrm{CsPbr}_{3}-\mathrm{CsPbCl}_{3} \mathrm{NWs}$, and (F, bottom) different patterns on anion-exchanged $\mathrm{CsPbBr} 3$ plates demonstrating the versatility of structures made using the current method. Blue emission: 410 to $450 \mathrm{~nm}$. Green emission: 500 to $550 \mathrm{~nm}$. Red emission: 580 to $640 \mathrm{~nm}$. (Scale bars for D, E, F, $3 \mu \mathrm{m}$.). (B) and (C) are adapted from Kong ${ }^{71}$. (D), (E), and (F) are adapted from Dou ${ }^{71}$.

The diverse range of photophysical properties arising from phase transitions and anion exchange have inspired the design of functional optoelectronic materials with sharp interfaces without using the conventional epitaxial growth methods. Utilizing a localized thermal-driven phase transition, we were able to control the transition completion in a single $\mathrm{LT}-\mathrm{CsSnI} 3 \mathrm{NW}$ and demonstrate $\mathrm{p}$-n junction formation within a LT-CsSnI $3 / \mathrm{HT}-\mathrm{CsSnI}_{3}$ two-phase heterojunction structure $^{71}$ (Figure 6B). As-synthesized LT-CsSnI 3 NWs show n-type conductivity, which is confirmed by a negative signals within Seebeck coefficient measurements. Upon thermal annealing, 
the Seebeck coefficient of HT-CsSnI 3 NWs becomes positive, confirming the electrical transition to p-type with the phase transition. To account for this, theoretical simulations suggest that a change from $\mathrm{V}_{\mathrm{I}}^{+}$to $\mathrm{V}_{\mathrm{Sn}}{ }^{2-}$ in the dominant defect-type results in a different majority carrier concentration between the two phases. CL microscopy with in-situ heating enables direct observation of the interface formation between them and clearly captures the directional interface propagation within a single NW (Figure 6B). As a result, the LT/HT-CsSnI 3 NW heterojunction exhibits current rectification, owing to the asymmetric p-n heterojunction properties (Figure 6C). Conventional p-n junctions are typically created by successive chemical doping processes and, thus, such phaseengineering of p-n junctions may enrich modern optical and electronic devices in halide perovskites. Further, the local nature of the heterojunction formed may act as fundamental building block toward high-density and large-scale applications.

By limiting anion exchange reactions to local regions on a single-crystalline nanomaterial using poly(methyl methacrylate) (PMMA) covers and electron beam lithography, we were able to demonstrate an effective way for directly obtaining halide perovskite semiconductor heterojunctions ${ }^{72}$. The two-segment or multiple heterojunctions between $\mathrm{CsPbCl}_{3}$ to $\mathrm{CsPbBr}_{3}$ or $\mathrm{CsPbI}_{3}$ to $\mathrm{CsPbBr}_{3}$ on single NWs displayed spatially resolved emissions ranging from red to blue with pixel sizes down to $500 \mathrm{~nm}$ (Figure 6D-F), confirming different local electronic structure. Such technological advances could be useful for miniaturised, high-density optoelectronic devices, like high resolution multicolour displays, large-scale integrated photonic devices or diode/transistor arrays.

\section{CONCLUSIONS AND OUTLOOK}

A paradigm exists within current halide perovskite research that "structure defines properties", however the question "what is the structure?" often remains open. Although it is a heavy task to fully understand the nature of phase transitions and anion exchange within halide perovskites, unlocking technologically relevant insights are likely to lead to important advances for the field. As such, this Account aimed to explore the origins of polymorphism and anion exchange phenomena within metal halide perovskites.

When the soft perovskite lattice diverges from normal equilibrium conditions, i.e., what one might consider the bulk properties, the phase energetics are shown to be inherently altered and may 
in fact be tailored toward more stable room temperature perovskite structures. On the other hand, environmental triggers, like moisture exposure, can act to destabilize its dynamic surface and catalyses a non-perovskite phase transition pathway which is mediated by an amorphous interfacial boundary. Ultimately, researchers need to isolate which aspects of (post)synthetic processing are key enablers, and inhibitors, for developing device-ready materials. For example, while the creation of nanocrystals and targeted surface functionalization can improve long-term phase stability, they can also impede charge carrier transport ${ }^{73}$ and the overall modified "big picture" must then be considered wisely.

As emphasised by several examples throughout this Account, the post-synthetic treatment of halide perovskite nano- and micro-crystals using local anion exchange and thermal-phase changes makes for a scientifically interesting toolbox. For instance, due to the intrinsic dependence of their optical properties on structure and composition, in situ luminescence microscopy has done well to not only visualize the transformation kinetics, but also quantify them. In the end, in situ characterisation methods have proven invaluable and have directly led to more comprehensive depictions of the dynamic halide perovskite crystal structure. For a versatile range of optoelectronic functions, flexible expressions of both phase and composition, down to the nanoscale, has inspired several lines of exciting applied research. Most notably, the realization of thermochromic windows, P-N junctions and high-density heterojunction arrays. We anticipate that shining a light on these topics will help broaden the knowledge base from which the field can explore and build interesting technologies moving forward.

\section{AUTHOR INFORMATION}

\section{Corresponding Authors}

*E-mail: julian.steele@kuleuven.be

*E-mail: p_yang@berkeley.edu

\section{Notes}

The authors declare no competing financial interest. 


\section{Biographies}

Julian A. Steele received his Ph.D. in physics from The Institute for Superconducting and Electronic Materials (ISEM), University of Wollongong, before joining the group of Prof. Roeffaers (KU Leuven) as a postdoctoral researcher with financial support from the Belgium government (FWO), where his work focuses on nanoscale optical materials. From 2019-2020, he undertook a postdoctoral research stay in the laboratory of Prof. Peidong Yang, at UC Berkeley, to work on phase transition phenomena within metal halide perovskites.

Minliang Lai received Ph.D. in Chemistry at University of California, Berkeley, under the supervision of Prof. Peidong Yang in 2019. His PhD research focuses on the phase transition and ion transport in halide perovskite nanomaterials. He is currently a postdoctoral scholar in Department of chemistry at Northwestern University.

Ye Zhang received her B.S. in Materials Chemistry from University of Science and Technology of China (USTC) in 2016. She is currently a Ph.D. Candidate in Prof. Peidong Yang's group at University of California, Berkeley. Her research focuses on studying the structural dynamics and symmetry-dictated properties in halide perovskites for optoelectronic and electromechanical applications.

Zhenni Lin received her B.S. in Materials Science and Engineering (MSE) from Rutgers University. She is currently an MSE Ph.D. candidate in Prof. Peidong Yang's group at University of California, Berkeley. Her work focuses on understanding and quantifying the impact of environmental stressors on phase transition of halide perovskites.

Johan Hofkens graduated from the Laboratory of Photochemistry and Spectroscopy, KU Leuven (Belgium), in 1993 studying TICT-states in organic molecules by time-resolved fluorescence measurements. After a postdoctoral stay (1994-1995) with Prof. Masuhara at Osaka University (Japan) on the development of optical trapping and with the late Prof. Barbara at University of Minneapolis (United States) on single-molecule spectroscopy, he returned to the KU Leuven were he started the single-molecule group and since 2020 he is a Max Plank Institute Fellow in Mainz, 
focusing on the development of optical microscopy tools to study challenging topics at the boundary of chemistry, biology, and physics.

Maarten B. J. Roeffaers graduated from the Centre for Surface Chemistry and Catalysis, KU Leuven (Belgium), in 2008 studying zeolite catalysis with fluorescence microscopy. After a postdoctoral stay (2009-2010) with Prof. Xie at Harvard University (United States) on the development and use of coherent Raman microscopy, he returned to the KU Leuven. In 2010, he started his own research group (www.roeffaers-lab.org) focusing on the development of optical microscopy tools to study heterogeneous catalysis and optically active materials such as Ag-zeolites and metal-halide perovskites.

Peidong Yang received a B.S. in chemistry from University of Science and Technology of China in 1993 and a Ph.D. in Chemistry from Harvard University in 1997. He did postdoctoral research at University of California, Santa Barbara, before joining the faculty in the Department of Chemistry at the University of California, Berkeley in 1999. He is currently professor in the Department of Chemistry, Materials Science and Engineering and a senior faculty scientist at the Lawrence Berkeley National Laboratory. He is the S. K. and Angela Chan Distinguished Chair Professor in Energy. He is the director for California Research Alliance by BASF and the Kavli Energy Nanoscience Institute.

\section{ACKNOWLEDGMENTS}

J.A.S. acknowledges financial support from the Research Foundation - Flanders (FWO: grant No.'s 12Y7218N and V439819N). This work was supported by the U.S. Department of Energy, Office of Science, Office of Basic Energy Sciences, Materials Sciences and Engineering Division, under Contract DE-AC02-05-CH11231 within the Physical Chemistry of Inorganic Nanostructures Program (KC3103). M.L. and Y.Z. acknowledge the fellowship support from Suzhou Industrial Park. J.H and M.B.J.R acknowledge financial support from the Research Foundation - Flanders (FWO) through research projects (FWO Grant No's G098319N and ZW15_09-GOH6316) and from the Flemish government through long term structural funding Methusalem (CASAS2, Meth/15/04)

\section{REFERENCES}


1. Kojima, A., Teshima, K., Shirai, Y. \& Miyasaka, T. Organometal halide perovskites as visiblelight sensitizers for photovoltaic cells. Journal of the American Chemical Society 131, 60506051 (2009).

2. Kim, H.-S. et al. Lead Iodide Perovskite Sensitized All-Solid-State Submicron Thin Film Mesoscopic Solar Cell with Efficiency Exceeding 9\%. Scientific Reports 2, srep00591 (2012).

3. Lee, M. M., Teuscher, J., Miyasaka, T., Murakami, T. N. \& Snaith, H. J. Efficient hybrid solar cells based on meso-superstructured organometal halide perovskites. Science 338, 643-647 (2012).

4. Snaith, H. J. Perovskites: The emergence of a new era for low-cost, high-efficiency solar cells. Journal of Physical Chemistry Letters 4, 3623-3630 (2013).

5. Kovalenko, M. V., Protesescu, L. \& Bodnarchuk, M. I. Properties and potential optoelectronic applications of lead halide perovskite nanocrystals. Science 358, 745-750 (2017).

6. Green, M. A. et al. Solar cell efficiency tables (version 51). Progress in Photovoltaics: Research and Applications 26, 3-12 (2018).

7. Christians, J. A., Habisreutinger, S. N., Berry, J. J. \& Luther, J. M. Stability in Perovskite Photovoltaics: A Paradigm for Newfangled Technologies. ACS Energy Letters 3, 2136-2143 (2018).

8. Yang, Y. \& You, J. Make perovskite solar cells stable. Nature 544, 155-156 (2017).

9. Christians, J. A. et al. Transformation of the excited state and photovoltaic efficiency of $\mathrm{CH} 3 \mathrm{NH} 3 \mathrm{PbI} 3$ perovskite upon controlled exposure to humidified air. Journal of the American Chemical Society 137, 1530-8 (2015).

10. Conings, B. et al. Intrinsic Thermal Instability of Methylammonium Lead Trihalide Perovskite. Advanced Energy Materials 5, (2015).

11. Eperon, G. E. et al. Formamidinium lead trihalide: a broadly tunable perovskite for efficient planar heterojunction solar cells. Energy \& Environmental Science 7, 982 (2014).

12. Wang, Y. et al. Thermodynamically stabilized $\beta-\mathrm{CsPI}_{3}$-based perovskite solar cells with efficiencies $>18 \%$. Science 365, 591-595 (2019). 
13. Kulbak, M., Cahen, D. \& Hodes, G. How Important Is the Organic Part of Lead Halide Perovskite Photovoltaic Cells? Efficient CsPbBr3 Cells. Journal of Physical Chemistry Letters 6, 2452-2456 (2015).

14. Kulbak, M. et al. Cesium Enhances Long-Term Stability of Lead Bromide Perovskite-Based Solar Cells. Journal of Physical Chemistry Letters 7, 167-172 (2016).

15. J., S. R. et al. Bandgap-Tunable Cesium Lead Halide Perovskites with High Thermal Stability for Efficient Solar Cells. Advanced Energy Materials 6, 1502458 (2016).

16. Eperon, G. E. et al. Inorganic caesium lead iodide perovskite solar cells. J. Mater. Chem. A 3, 19688-19695 (2015).

17. Whitfield, P. S. et al. Structures, Phase Transitions and Tricritical Behavior of the Hybrid Perovskite Methyl Ammonium Lead Iodide. Sci Rep 6, 35685 (2016).

18. Swainson, I. P., Hammond, R. P., Soullière, C., Knop, O. \& Massa, W. Phase transitions in the perovskite methylammonium lead bromide, CH3ND3PbBr3. Journal of Solid State Chemistry 176, 97-104 (2003).

19. Xu, Q., Eguchi, T., Nakayama, H., Nakamura, N. \& Kishita, M. Molecular Motions and Phase Transitions in Solid CH3NH3PbX3 (X = C1, Br, I) as Studied by NMR and NQR. Zeitschrift für Naturforschung A 46, (1991).

20. Fabini, D. H. et al. Reentrant Structural and Optical Properties and Large Positive Thermal Expansion in Perovskite Formamidinium Lead Iodide. Angew. Chem. Int. Ed. 55, 15392-15396 (2016).

21. Schueller, E. C. et al. Crystal Structure Evolution and Notable Thermal Expansion in Hybrid Perovskites Formamidinium Tin Iodide and Formamidinium Lead Bromide. Inorg. Chem. 57, 695-701 (2018).

22. Govinda, S. et al. Critical Comparison of $\mathrm{FAPbX}_{3}$ and $\mathrm{MAPbX}_{3}(\mathrm{X}=\mathrm{Br}$ and $\mathrm{Cl})$ : How Do They Differ? J. Phys. Chem. C 122, 13758-13766 (2018).

23. Marronnier, A. et al. Anharmonicity and Disorder in the Black Phases of Cesium Lead Iodide Used for Stable Inorganic Perovskite Solar Cells. ACS Nano 12, 3477-3486 (2018). 
24. Hirotsu, S., Harada, J., Iizumi, M. \& Gesi, K. Structural Phase Transitions in $\mathrm{CsPbBr}_{3}$. J. Phys. Soc. Jpn. 37, 1393-1398 (1974).

25. Plesko, S., Kind, R. \& Roos, J. Structural Phase Transitions in $\mathrm{CsPbCl}_{3}$ and $\mathrm{RbCdCl}_{3}$. J. Phys. Soc. Jpn. 45, 553-557 (1978).

26. Chung, I. et al. CsSnI 3 : Semiconductor or Metal? High Electrical Conductivity and Strong Near-Infrared Photoluminescence from a Single Material. High Hole Mobility and PhaseTransitions. J. Am. Chem. Soc. 134, 8579-8587 (2012).

27. Fabini, D. H. et al. Dynamic Stereochemical Activity of the $\mathrm{Sn}^{2+}$ Lone Pair in Perovskite CsSnBr 3. J. Am. Chem. Soc. 138, 11820-11832 (2016).

28. Huang, L. \& Lambrecht, W. R. L. Lattice dynamics in perovskite halides CsSn X 3 with X = I, Br, Cl. Phys. Rev. B 90, 195201 (2014).

29. Yashima, M. \& Ali, R. Structural phase transition and octahedral tilting in the calcium titanate perovskite CaTiO3. Solid State Ionics 180, 120-126 (2009).

30. Glazer, A. M. The classification of tilted octahedra in perovskites. Acta Crystallogr B Struct Crystallogr Cryst Chem 28, 3384-3392 (1972).

31. Howard, C. J. \& Stokes, H. T. Group-Theoretical Analysis of Octahedral Tilting in Perovskites. Erratum. Acta Crystallogr B Struct Sci 58, 565-565 (2002).

32. Steele, J. A. et al. Thermal unequilibrium of strained black $\mathrm{CsPbI}_{3}$ thin films. Science $\mathbf{3 6 5}$, 679-684 (2019).

33. Shannon, R. D. Revised effective ionic radii and systematic studies of interatomic distances in halides and chalcogenides. Acta Cryst A 32, 751-767 (1976).

34. Kieslich, G., Sun, S. \& Cheetham, A. K. Solid-state principles applied to organic-inorganic perovskites: new tricks for an old dog. Chem. Sci. 5, 4712-4715 (2014).

35. van der Stam, W. et al. Highly Emissive Divalent-Ion-Doped Colloidal $\mathrm{CsPb}{ }_{1-x} \mathrm{M}_{x} \mathrm{Br}_{3}$ Perovskite Nanocrystals through Cation Exchange. J. Am. Chem. Soc. 139, 4087-4097 (2017).

36. Bartel, C. J. et al. New tolerance factor to predict the stability of perovskite oxides and halides. Sci. Adv. 5, eaav0693 (2019). 
37. Goldschmidt, V. M. Die Gesetze der Krystallochemie. Naturwissenschaften 14, 477-485 (1926).

38. Green, M. A., Ho-Baillie, A. \& Snaith, H. J. The emergence of perovskite solar cells. Nat Photon 8, 506-514 (2014).

39. Stoumpos, C. C., Malliakas, C. D. \& Kanatzidis, M. G. Semiconducting tin and lead iodide perovskites with organic cations: Phase transitions, high mobilities, and near-infrared photoluminescent properties. Inorganic Chemistry 52, 9019-9038 (2013).

40. Onoda-Yamamuro, N., Matsuo, T. \& Suga, H. Calorimetric and IR spectroscopic studies of phase transitions in methylammonium trihalogenoplumbates (II) †. Journal of Physics and Chemistry of Solids 51, 1383-1395 (1990).

41. Saliba, M. et al. Incorporation of rubidium cations into perovskite solar cells improves photovoltaic performance. Science 354, 206-209 (2016).

42. Howard, C. J. \& Stokes, H. T. Structures and phase transitions in perovskites - a grouptheoretical approach. Acta Cryst. A61, 93-111 (2005).

43. Howard, C. J., Knight, K. S., Kennedy, B. J. \& Kisi, E. H. The structural phase transitions in strontium zirconate revisited. J. Phys.: Condens. Matter 12, L677-L683 (2000).

44. Sutton, R. J. et al. Bandgap-Tunable Cesium Lead Halide Perovskites with High Thermal Stability for Efficient Solar Cells. Advanced Energy Materials 6, (2016).

45. Protesescu, L. et al. Nanocrystals of Cesium Lead Halide Perovskites $\left(\mathrm{CsPbX}_{3}, \mathrm{X}=\mathrm{Cl}, \mathrm{Br}\right.$, and I): Novel Optoelectronic Materials Showing Bright Emission with Wide Color Gamut. Nano Lett. 15, 3692-3696 (2015).

46. Dastidar, S. et al. Quantitative Phase-Change Thermodynamics and Metastability of PerovskitePhase Cesium Lead Iodide. J. Phys. Chem. Lett. 8, 1278-1282 (2017).

47. Frolova, L. A. et al. Highly efficient all-inorganic planar heterojunction perovskite solar cells produced by thermal coevaporation of CsI and PbI2. Journal of Physical Chemistry Letters $\mathbf{8}$, 67-72 (2017). 
48. Burwig, T., Fränzel, W. \& Pistor, P. Crystal Phases and Thermal Stability of Co-evaporated $\mathrm{CsPbX}_{3}(\mathrm{X}=\mathrm{I}, \mathrm{Br})$ Thin Films. The Journal of Physical Chemistry Letters 9, 4808-4813 (2018).

49. Zhao, Q. et al. Size-Dependent Lattice Structure and Confinement Properties in $\mathrm{CsPbI} 3$ Perovskite Nanocrystals: Negative Surface Energy for Stabilization. ACS Energy Lett. 5, 238247 (2020).

50. Zhao, B. et al. Thermodynamically Stable Orthorhombic $\gamma$-CsPbI 3 Thin Films for HighPerformance Photovoltaics. Journal of the American Chemical Society 140, 11716-11725 (2018).

51. Fu, Y. et al. Stabilization of the Metastable Lead Iodide Perovskite Phase via Surface Functionalization. Nano Letters 17, 4405-4414 (2017).

52. Jain, S. C., Willander, M. \& Overstraeten, R. van. Compound semiconductors strained layers and devices. (Kluwer Academic Publishers, 2000).

53. Rabe, K. M. \& Waghmare, U. V. Strain coupling in perovskite structural transitions: A first principles approach. Ferroelectrics 194, 119-134 (1997).

54. Granger, P., Parvulescu, V. I., Kaliaguine, S. \& Prellier, W. Perovskites and Related Mixed Oxides: Concepts and Applications. (2015). doi:10.1002/9783527686605.

55. Zhao, J. et al. Strained hybrid perovskite thin films and their impact on the intrinsic stability of perovskite solar cells. Sci. Adv. 3, eaao5616 (2017).

56. He, F. et al. Structural phase transition in epitaxial perovskite films. Physical Review B Condensed Matter and Materials Physics 70, 1-10 (2004).

57. Lin, J. et al. Thermochromic halide perovskite solar cells. Nature Mater 17, 261-267 (2018).

58. Connor G. Bischak and Minliang Lai and Dylan Lu and Zhaochuan Fan and Philippe David and Dengpan Dong and Hong Chen and Ahmed S. Etman and Teng Lei and Junliang Sun and Michael Grünwald and David T. Limmer and Peidong Yang and Naomi S. Ginsber. LiquidLike Interfaces Mediate Structural Phase Transitions in Lead Halide Perovskite. arXiv 1907.13509 (2019). 
59. Lei, T. et al. Electrical and Optical Tunability in All-Inorganic Halide Perovskite Alloy Nanowires. Nano Lett. 18, 3538-3542 (2018).

60. Yu, Y. et al. Atomic Resolution Imaging of Halide Perovskites. Nano Lett. 16, 7530-7535 (2016).

61. Keshavarz, M. et al. Tracking Structural Phase Transitions in Lead-Halide Perovskites by Means of Thermal Expansion. Adv. Mater. 1900521 (2019) doi:10.1002/adma.201900521.

62. Chen, H. et al. Structural and spectral dynamics of single-crystalline Ruddlesden-Popper phase halide perovskite blue light-emitting diodes. Sci. Adv. 6, eaay4045 (2020).

63. Lin, J. et al. Pressure-induced semiconductor-to-metal phase transition of a charge-ordered indium halide perovskite. Proc Natl Acad Sci USA 116, 23404-23409 (2019).

64. Lai, M. et al. Intrinsic anion diffusivity in lead halide perovskites is facilitated by a soft lattice. Proc Natl Acad Sci USA 115, 11929-11934 (2018).

65. Zhang, Y. et al. Quantitative imaging of anion exchange kinetics in halide perovskites. Proc Natl Acad Sci USA 116, 12648-12653 (2019).

66. Koscher, B. A., Bronstein, N. D., Olshansky, J. H., Bekenstein, Y. \& Alivisatos, A. P. Surfacevs Diffusion-Limited Mechanisms of Anion Exchange in $\mathrm{CsPbBr}_{3}$ Nanocrystal Cubes Revealed through Kinetic Studies. J. Am. Chem. Soc. 138, 12065-12068 (2016).

67. Zhang, D., Eaton, S. W., Yu, Y., Dou, L. \& Yang, P. Solution-Phase Synthesis of Cesium Lead Halide Perovskite Nanowires. J. Am. Chem. Soc. 137, 9230-9233 (2015).

68. Zhang, D. et al. Synthesis of Composition Tunable and Highly Luminescent Cesium Lead Halide Nanowires through Anion-Exchange Reactions. J. Am. Chem. Soc. 138, 7236-7239 (2016).

69. Steele, J. A. et al. Direct Laser Writing of $\delta$ - to $\alpha$-Phase Transformation in Formamidinium Lead Iodide. ACS Nano 11, 8072-8083 (2017).

70. Pisoni, A. et al. Ultra-low thermal conductivity in organic-inorganic hybrid perovskite CH3NH3PbI3. Journal of Physical Chemistry Letters 5, 2488-2492 (2014). 
71. Kong, Q. et al. Phase-transition-induced p-n junction in single halide perovskite nanowire. Proc Natl Acad Sci USA 115, 8889-8894 (2018).

72. Dou, L. et al. Spatially resolved multicolor $\mathrm{CsPb}_{3}$ nanowire heterojunctions via anion exchange. Proc Natl Acad Sci USA 114, 7216-7221 (2017).

73. Sanehira, E. M. et al. Enhanced mobility $\mathrm{CsPbI}_{3}$ quantum dot arrays for record-efficiency, high-voltage photovoltaic cells. Sci. Adv. 3, eaao4204 (2017). 